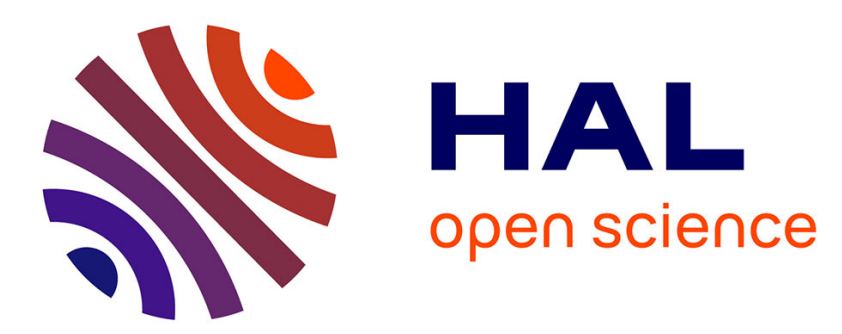

\title{
Identification of equivalent multipolar electromagnetic sources by spatial filtering
}

Benjamin Vincent, Olivier Chadebec, Jean-Luc Schanen, Kévin Berger, Ronan

Perrussel, Laurent Krähenbühl

\section{- To cite this version:}

Benjamin Vincent, Olivier Chadebec, Jean-Luc Schanen, Kévin Berger, Ronan Perrussel, et al.. Identification of equivalent multipolar electromagnetic sources by spatial filtering. IEEE Transactions on Magnetics, 2010, 46 (8), pp.2815 - 2818. 10.1109/TMAG.2010.2043421 . hal-00455455

\section{HAL Id: hal-00455455 https://hal.science/hal-00455455}

Submitted on 27 Jul 2010

HAL is a multi-disciplinary open access archive for the deposit and dissemination of scientific research documents, whether they are published or not. The documents may come from teaching and research institutions in France or abroad, or from public or private research centers.
L'archive ouverte pluridisciplinaire HAL, est destinée au dépôt et à la diffusion de documents scientifiques de niveau recherche, publiés ou non, émanant des établissements d'enseignement et de recherche français ou étrangers, des laboratoires publics ou privés. 


\title{
Identification of Equivalent Multipolar Electromagnetic Sources by Spatial Filtering
}

\author{
Benjamin Vincent ${ }^{1,2}$, Olivier Chadebec ${ }^{2}$, Jean-Luc Schanen ${ }^{2}$, Kévin Berger ${ }^{1}$, Ronan Perrussel ${ }^{1}$, and \\ Laurent Krähenbühl ${ }^{1}$ \\ ${ }^{1}$ Laboratoire Ampère (CNRS UMR5005), Université de Lyon, École Centrale de Lyon, Ecully 69134, France \\ ${ }^{2}$ Grenoble Electrical Engineering Lab (CNRS UMR5269), Université Joseph Fourier and Grenoble INP, ENSEEE, Grenoble \\ 38402, France
}

\begin{abstract}
New sensors of magnetic induction in near field, dedicated to studies of electromagnetic compatibility, are proposed based on the Standard CISPR16-1 coils. The new coil shape allows the sensors to be sensitive only to specific components of the multipolar expansion, which is similar to a spatial filtering. In comparison with our previous approach in which we designed a complete set of coils, we aim to simplify the sensor coil geometry by rotating the spherical harmonics functions. After a description of the tools required, the design method is described.
\end{abstract}

Index Terms-Electromagnetic compatibility, identification, magnetic field measurement, multipolar expansion, power electronics, transducers.

\section{INTRODUCTION}

$\mathbf{F}$ OR electromagnetic compatibility studies, a reduced model of the stray magnetic field generated by power electronic systems diminishes design cost. To determine this model, our work focuses on an experimental approach based on the use of large loop antennas. These antennas offer advantages, such as reducing the effect of positioning inaccuracies and reducing measurement time in comparison with punctual measurement systems. For instance, to identify the first order of a multipolar expansion (dipole) [1], the Standard CISPR 16-1 [2] uses three orthogonal loops (Fig. 1). This approach works for the medium frequency range: $9 \mathrm{kHz}$ to $30 \mathrm{MHz}$.

The problem is that no source is composed of pure dipoles. Therefore, even the standard method can lead to errors. In a previous work, we proposed accounting for higher order terms in the expansion by using an original coil sensor design to improve accuracy [3]. Here, we introduce a simplification of the sensor design. The principle of this design and the improvements are reviewed.

\section{MULTIPOLAR EXPANSION}

For near-field studies in the standard frequency range $(9 \mathrm{kHz}$ to $30 \mathrm{MHz}$ ), a quasi-static approximation is suitable. Outside a validity sphere, defined as the minimum area of the expansion convergence and including all radiation sources, the magnetic field can be described by the magnetic scalar potential $\psi(t)$ solution of the Laplace equation at each instant. Using the Fourier transform in time leads us to consider a potential $\Psi$ satisfying the Laplace equation for each frequency. For each of these frequencies, in a given spherical coordinate system and in the near-field area of the source, the solution can be written using a multipolar expansion

Manuscript received December 23, 2009; accepted February 08, 2010. Current version published July 21, 2010. Corresponding author: B. Vincent (e-mail: benigor.vincent@free.fr).

Color versions of one or more of the figures in this paper are available online at http://ieeexplore.ieee.org.

Digital Object Identifier 10.1109/TMAG.2010.2043421

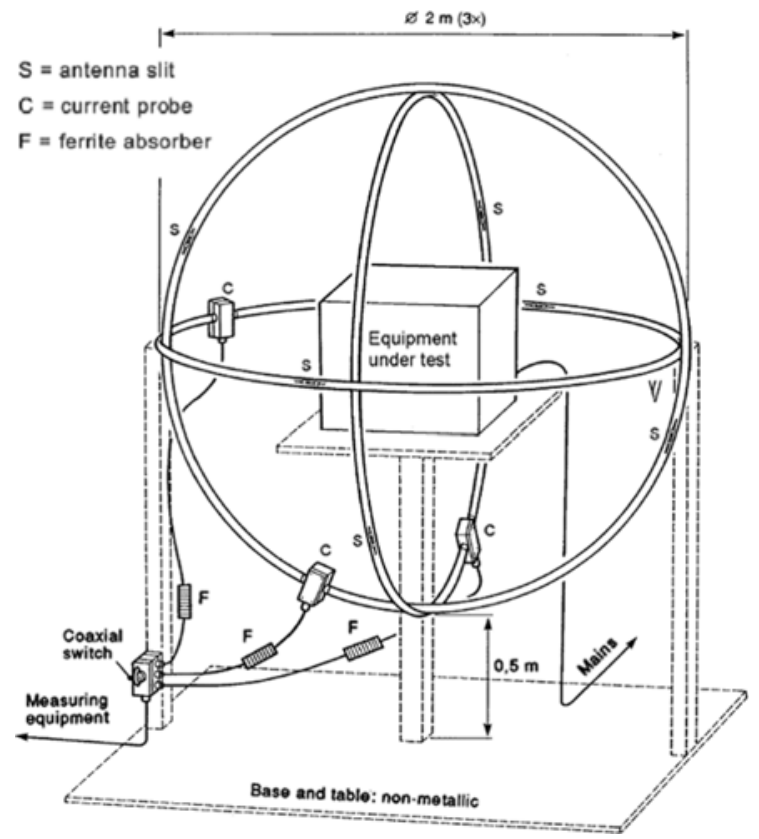

Fig. 1. Large Loop Antenna (LLA) system from the Standard CISPR 16-1 consisting of three orthogonal loop-antennas [2].

$\mathbf{B}=-\mu_{0} \vec{\nabla} \Psi$ with:

$$
\Psi(r, \theta, \varphi)=\frac{1}{4 \pi} \sum_{n=1}^{+\infty} \sum_{m=-n}^{+n} A_{n m} \frac{1}{r^{n+1}} S_{n m}(\theta, \varphi)
$$

where $S_{n m}$ are the real spherical harmonic functions of order $n$ and degree $m,(r, \theta, \varphi)$ are the spherical coordinates whose origin is the center of expansion, and $A_{n m}$ are the unknown coefficients. A hierarchy of the expansion terms based on the order $n$, for which the amplitude of the terms decreases as $1 / r^{n+2}$, makes a truncated version of (1) a relevant reduced model for studying distant interactions between power electronic systems.

\section{SENSOR DESIGN}

The measurement principle is similar to a spatial filtering: According to the coil shape, each sensor is sensitive to its specific component of the multipolar expansion. 


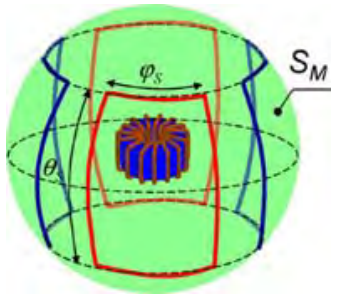

Fig. 2. Example of a component sensor located on sphere $S_{M}$ and centered on the device under test. The sensor is composed of several coils defined by angles $\theta_{S}$ and $\varphi_{S}$.

\section{A. Preliminaries}

Each component sensor, dedicated to a specific component of the multipolar expansion, is constituted of several coils. They are located on a sphere $S_{M}$, with a radius $r_{M}$, including the validity sphere region. An example is shown in Fig. 2.

The principle consists in measuring the magnetic flux through the coils. As a component sensor is constituted of several coils, it is possible to total all flux (sum or difference). The following representation will be used in this paper: A red coil will correspond to a positive outgoing flux, whereas a blue one will correspond to a negative outgoing flux (see Fig. 2).

Any set of coils on the sphere $S_{M}$ can be represented by a "sensor function" $C$ defined by:

$-C(\theta, \varphi)=1$ for a portion of $S_{M}$ delimited by the red coil;

$-C(\theta, \varphi)=-1$ for a portion of $S_{M}$ delimited by the blue coil;

$-C(\theta, \varphi)=0$ for the remaining $S_{M}$ area.

In the following, let $n$ and $m$ denote the indices of the component $A_{n m}$ to be identified and $C^{n m}$ the "sensor function" dedicated to $\mathbf{B}_{n m}=-\left(\mu_{0} / 4 \pi\right) \cdot \vec{\nabla}\left(A_{n m} r^{-(n+1)} S_{n m}(\theta, \varphi)\right)$. According to the radial component of $\mathbf{B}$, written as

$$
B_{r}\left(r_{M}, \theta, \varphi\right)=\frac{\mu_{0}}{4 \pi} \sum_{n^{\prime}=1}^{+\infty} \sum_{m^{\prime}=-n^{\prime}}^{+n^{\prime}} \frac{\left(n^{\prime}+1\right)}{r_{M}^{n^{\prime}+2}} A_{n^{\prime} m^{\prime}} S_{n^{\prime} m^{\prime}}(\theta, \varphi)
$$

the measured flux through sensor function $C^{n m}$ corresponds to

$$
\begin{aligned}
\text { Flux }_{n m}= & \iint_{S_{M}} C^{n m}(\theta, \varphi) B_{r}\left(r_{M}, \theta, \varphi\right) d s \\
= & \frac{\mu_{0}}{4 \pi} \sum_{n^{\prime}=1}^{+\infty} \frac{\left(n^{\prime}+1\right)}{r_{M}^{n^{\prime}+2}} \sum_{m^{\prime}=-n^{\prime}}^{+n^{\prime}} A_{n^{\prime} m^{\prime}} \\
& \times\left(C_{n^{\prime} m^{\prime}}^{n m} \iint_{S_{M}}\left|S_{n^{\prime} m^{\prime}}\right|^{2} d s\right)
\end{aligned}
$$

where $C_{n^{\prime} m^{\prime}}^{n m}$ are the coefficients of the spherical harmonics decomposition of the $C^{n m}$ function

$$
C^{n m}(\theta, \varphi)=\sum_{n^{\prime}=1}^{+\infty} \sum_{m^{\prime}=-n^{\prime}}^{+n^{\prime}} C_{n^{\prime} m^{\prime}}^{n m} S_{n^{\prime} m^{\prime}}(\theta, \varphi) .
$$

These coefficients are calculated as follows:
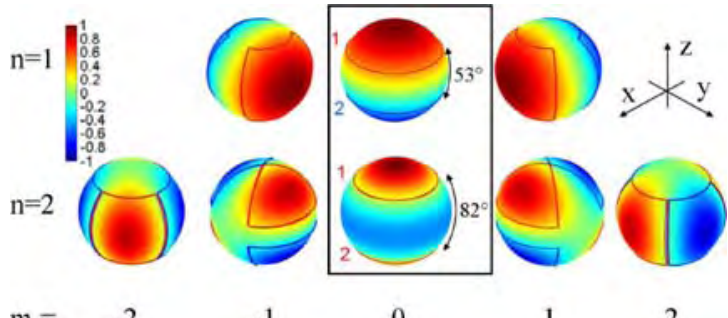

$\mathrm{m}=-2$

$-1$

2

Fig. 3. Eight coil sensors for the identification of the first and second orders The flux is positive for the red coils and negative for the blue ones. Functions $S_{n m}$ are projected on the measurement sphere.

$$
C_{n^{\prime} m^{\prime}}^{n m}=\frac{\iint_{S_{M}} C^{n m} S_{n^{\prime} m^{\prime}} d s}{\iint_{S_{M}}\left|S_{n^{\prime} m^{\prime}}\right|^{2} d s}=\frac{\left(2 n^{\prime}+1\right)}{4 \pi r_{M}^{2}} \iint_{S_{M}} C^{n m} S_{n^{\prime} m^{\prime}} d s .
$$

Ideally, the particular $A_{n m}$ to be identified with $C^{n m}$ sensor function could be written as

$$
C_{n m}^{n m} \neq 0 \quad \text { and } \quad C_{n^{\prime} m^{\prime}}^{n m}=0 \quad \forall\left(n^{\prime}, m^{\prime}\right) \neq(n, m) .
$$

While, according to (4), $C^{n m}$ and $S_{n m}$ are proportional, this assumption (6) is incompatible with the $C^{n m}$ definition. However, if multipolar expansion of the source is limited to the fourth order, then solutions exist. In a previous work [3], we have proven that it was possible to design coils such that

$$
\left\{\begin{array}{l}
C_{n^{\prime} m^{\prime}}^{n m} \neq 0 \text { for }\left\{\begin{array} { l } 
{ n ^ { \prime } = n } \\
{ m ^ { \prime } = m } \\
{ C _ { n ^ { \prime } m ^ { \prime } } ^ { n m } = 0 }
\end{array} \text { for } \left\{\begin{array}{l}
n^{\prime} \neq n \\
m^{\prime} \neq m
\end{array} \quad \forall\left(n^{\prime} \in[1 ; 4] ; m^{\prime} \in\left[-n^{\prime} ; n^{\prime}\right]\right) .\right.\right.
\end{array}\right.
$$

In other words, we proposed a system of coils that allowed the identification of the first two orders ( $n=1$ and 2 ) by filtering the two orders after them ( $n=3$ and 4 ). Consequently, if the multipolar expansion of the source is limited to the fourth order, our sensor should theoretically provide the dipolar and quadrupolar coefficients without error. If higher orders $\left(n^{\prime}>4\right)$ are present, they introduce some error, but their influence decreases at least by $1 / r^{5+2}$. In our study, we will consider that the multipolar expansion is limited to the fourth order. This is considered sufficient for most practical power electronic radiating systems. This work has resulted in a design with eight flux coil sensors located on the measurement sphere. The eight sensors are presented in Fig. 3. These first shapes are linked to spherical coordinates outlined within the $\theta$ or $\varphi$ constant. Unfortunately, this design is too complex to actually build.

\section{B. Rotational Properties of $S_{n^{\prime} m^{\prime}}$}

We wanted to simplify this solution in order to build a practical sensor. The basic idea comes from the properties of spherical harmonic functions: By rotating and summing only one $S_{n m}$ component, it is possible to produce all components of the same $n$ order. Examples are given in Fig. 4. For the first-order rotation, the shapes of the components are naturally the same regardless of the $\pi / 2$ rotation. The second-order case is more complex: one $\pi / 4$ rotation around the $y$-axis and one $\pi / 2$ rotation around the x-axis of the $S_{21}$ component generates the $S_{22}$ component. The idea is that it is possible to generalize this property to design sensors. Thus, to simplify the design, we must 


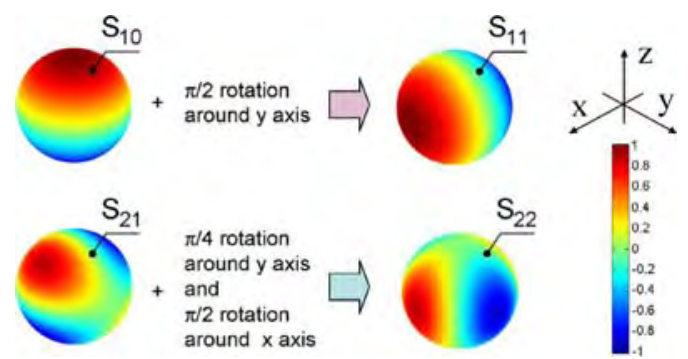

Fig. 4. Rotation properties of spherical harmonics. At the $n$ order, only one shape is necessary in order to create all others.
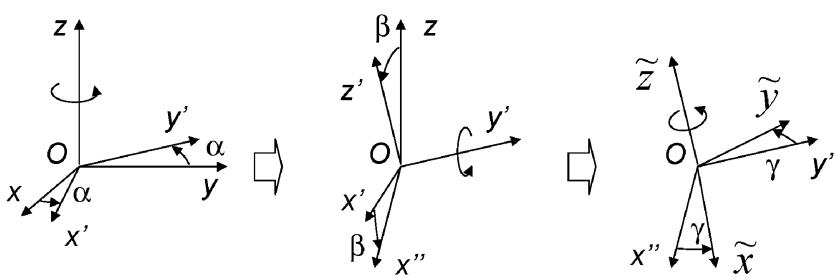

Fig. 5. Rotation description using Euler angles $(\alpha, \beta, \gamma)$.

start from simpler sensor coils, i.e., with the $C^{10}$ and $C^{20}$ sensors as coplanar coils, shown in the black frame in Fig. 3.

In particular, from (3) and (5), we found relationships between the $A_{10}$ and $A_{20}$ components and their measured fluxes. These are given by

$$
\begin{aligned}
& A_{10}=\frac{10^{8} r_{M}}{32 \pi}\left(f l u x_{1}-\text { flux }_{2}\right) \\
& A_{20}=\frac{6125.10^{4} r_{M}^{2}}{3 \pi \sqrt{21}}\left(\text { flux }_{1}+\text { flux }_{2}\right)
\end{aligned}
$$

with the fluxes indicated in Fig. 3.

\section{Sensor Design}

1) Rotation Convention: In order to describe rotation, Euler angles are used as shown in Fig. 5. Any rotation is composed by three elementary rotations. The first elementary rotation is with the $\alpha$ angle around the $\mathrm{z}$-axis, the second one is with the $\beta$ angle around the $y^{\prime}$-axis, and the last one is with the $\gamma$ around the $\mathrm{z}^{\prime}$-axis. In the following section, this rotation is named $R(\alpha, \beta, \gamma)$, and the new coordinate system with this rotation is denoted $(O, \widetilde{x}, \widetilde{y}, \widetilde{z})$.

2) Rotation of the Spherical Harmonics: As $\left(S_{n^{\prime} m^{\prime}}\right)$ denotes the real spherical harmonics in the coordinate system $(O, x, y, z),\left(\widetilde{S}_{n^{\prime} m^{\prime}}\right)$ will denote the same spherical harmonics in the rotated coordinate system $(O, \widetilde{x}, \widetilde{y}, \widetilde{z})$.

For a fixed $n^{\prime}$, the functions $\left(S_{n^{\prime} m^{\prime}}\right)_{m^{\prime} \in\left\{-n^{\prime} ;+n^{\prime}\right\}}$ and $\left(\widetilde{S}_{n^{\prime} m^{\prime}}\right)_{m^{\prime} \in\left\{-n^{\prime} ;+n^{\prime}\right\}}$ are both orthogonal bases of the spherical harmonics of order $n^{\prime}$, and consequently a change of basis matrix $R^{\left(n^{\prime}\right)}$ can be defined such that

$$
\widetilde{S}_{n^{\prime} m^{\prime}}=\sum_{p=-n^{\prime}}^{n^{\prime}} R_{p, m^{\prime}}^{\left(n^{\prime}\right)} S_{n^{\prime} p} \quad \forall m^{\prime} \in\left[-n^{\prime} ; n^{\prime}\right] .
$$

In particular, we focus on the $\widetilde{S}_{10}$ and $\widetilde{S}_{20}$ components. $R^{(1)}$ and $R^{(2)}$ can be found, for instance, in [4], and the coefficients of $\widetilde{S}_{10}$ and $\widetilde{S}_{20}$, respectively, in the bases $\left(S_{1 m^{\prime}}\right)$ and $\left(S_{2 m^{\prime}}\right)$ are given by

$$
\begin{aligned}
& R^{(1)}\left[\begin{array}{l}
0 \\
1 \\
0
\end{array}\right]=\left[\begin{array}{c}
\sin (\alpha) \sin (\beta) \\
\cos (\beta) \\
\cos (\alpha) \sin (\beta)
\end{array}\right] \\
& R^{(2)}\left[\begin{array}{l}
0 \\
0 \\
1 \\
0 \\
0
\end{array}\right]=\left[\begin{array}{c}
\sqrt{3} / 2 \sin (2 \alpha) \sin ^{2}(\beta) \\
\sqrt{3} / 2 \sin (\alpha) \sin (2 \beta) \\
1 / 4(1+3 \cos (2 \beta) \\
\sqrt{3} / 2 \cos (\alpha) \sin (2 \beta) \\
\sqrt{3} / 2 \cos (2 \alpha) \sin ^{2}(\beta)
\end{array}\right] .
\end{aligned}
$$

We used $S_{10}^{\alpha, \beta}$ to denote the $\widetilde{S}_{10}$ harmonic component expressed in the coordinate system with $R(\alpha, \beta, \gamma$,$) rotation.$ From the first order $n=1$, from (11), we can deduce

$$
S_{10}^{0, \pi / 2}=S_{11} \quad \text { and } \quad S_{10}^{\pi / 2, \pi / 2}=S_{1,-1} .
$$

This explains the first rotational relationship in Fig. 4: All components of the first order could be created using only the rotation of $S_{10}$.

For the second order, from (12), we have

$$
\begin{aligned}
& S_{21}=\frac{1}{\sqrt{3}}\left(S_{20}^{0, \pi / 4}-S_{20}^{0,-\pi / 4}\right) \\
& S_{22}=\frac{1}{\sqrt{3}}\left(S_{20}^{0, \pi / 2}-S_{20}^{\pi / 2, \pi / 2}\right) .
\end{aligned}
$$

As for the first order, according to (13) and (14), certain rotations of $S_{20}$ could create all other second-order components. From components with $m^{\prime}>0$, we can deduce ones with $m^{\prime}<0$ using geometrical symmetries of spherical harmonics

$$
S_{n^{\prime} m^{\prime}}\left(\theta, \varphi+\pi /\left(2 m^{\prime}\right)\right)=-S_{n^{\prime},-m^{\prime}}(\theta, \varphi) .
$$

3) Rotation Effect on the Measurement: According to (4), if the $C^{n m}$ sensor function rotates, it keeps the same coefficients in the spherical harmonic decomposition for the new coordinate system as for the old coordinate system

$$
\begin{aligned}
C^{n m}(\theta, \varphi) & =\sum_{\left(n^{\prime}, m^{\prime}\right)} C_{n^{\prime} m^{\prime}}^{n m} \widetilde{S}_{n^{\prime} m^{\prime}}(\theta, \varphi) \\
& =\sum_{\left(n^{\prime}, m^{\prime}\right)} C_{n^{\prime} m^{\prime}}^{n m} \sum_{p=-n^{\prime}}^{n^{\prime}} R_{p, m^{\prime}}^{\left(n^{\prime}\right)} S_{n^{\prime} p}(\theta, \varphi) .
\end{aligned}
$$

Thus, the measured flux by $C^{n m}$ corresponds to

$$
\begin{aligned}
\text { Flux }_{n m} & =\frac{\mu_{0}}{4 \pi} \sum_{n^{\prime}=1}^{+\infty} \frac{\left(n^{\prime}+1\right)}{r_{M}^{n^{\prime}+2}} \sum_{m^{\prime}=-n^{\prime}}^{+n^{\prime}}\left(A_{n^{\prime} m^{\prime}}\right. \\
& \left.\times\left(\iint_{S_{M}}\left|S_{n^{\prime} m^{\prime}}(\theta, \varphi)\right|^{2}\right) \sum_{p=-n^{\prime}}^{+n^{\prime}} R_{m^{\prime}, p}^{\left(n^{\prime}\right)} C_{n^{\prime} p}^{n m}\right) .
\end{aligned}
$$

In consequence, if the sensor is rotated, other components $\mathbf{B}_{n m}$ could create disturbances, but all of them will be from the same order $n$. Yet, the sensor set designs in Fig. 3 are insensitive to disturbances above the fourth order. The filtering capability is still maintained for any sensor rotation.

\section{Example of $C^{21}$ Sensor Design}

From (13), the $S_{21}$ function is generated from the rotational and linear combination of $S_{20}$. Thus, as shown in Fig. 6, the $C^{21}$ sensor is made by using the $C^{20}$ sensors associated with two 


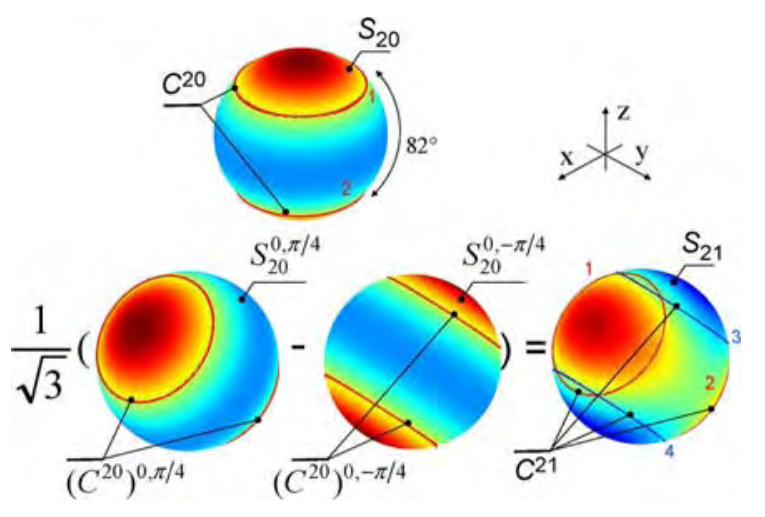

Fig. 6. Identification of the $A_{21}$ parameter using $C^{20}$ sensor topology and associated rotation and flux combinations.

rotations and a linear combination. We denote $\left(C^{n m}\right)^{\alpha, \beta}$ as the $C^{n m}$ sensor in the rotated system. Thus, according to Fig. 6, we have

$$
C^{21}=\left(C^{20}\right)^{0, \pi / 4}-\left(C^{20}\right)^{0,-\pi / 4}
$$

According to (7) and (13), it is equivalent to

$$
\begin{aligned}
C^{21} & =C_{20}^{20} S^{0, \pi / 4}-C_{20}^{20} S^{0,-\pi / 4}+\text { terms with } n^{\prime}>4 \\
& =\sqrt{3} C_{20}^{20} S_{21}+\text { terms with } n^{\prime}>4 .
\end{aligned}
$$

Thus, on one hand, we have

$$
\text { Flux }_{21}=\text { flux }_{1}+\text { flux }_{2}-f l u x_{3}-\text { flux }_{4}
$$

where $\operatorname{flux}_{i}$ corresponds to the flux measured across the coil $i$ in Fig. 6; on the other hand, according to (3) and (19)

$$
\begin{aligned}
\text { Flux }_{21} & =\iint_{S_{M}} C^{21} B_{r} d s=\sqrt{3} C_{20}^{20} \iint_{S_{M}} S_{21} B_{r} d s \\
& =\sqrt{3} C_{20}^{20} \frac{\mu_{0}}{4 \pi} \frac{(2+1)}{r_{M}^{2+2}} A_{21} \iint_{S_{M}}\left|S_{21}\right|^{2} d s
\end{aligned}
$$

where the higher order terms are null because of the assumption that the multipolar expansion is limited to the order 4. Finally, we obtain

$$
\begin{aligned}
A_{21} & =\frac{4 \pi}{\mu_{0}} \frac{r_{M}^{4}}{3} \frac{1}{C_{20}^{20}}\left(\frac{1}{\sqrt{3}}\right) \cdot \text { Flux }_{21} \\
& =\frac{6125 \cdot 10^{4} r_{M}^{2}}{3 \pi \sqrt{21}}\left(\frac{1}{\sqrt{3}}\right) \cdot \text { Flux }_{21} .
\end{aligned}
$$

\section{E. Final Topology}

We present in Fig. 7 the simplification of the shapes obtained using the method based on rotations. For the first order, the $C^{10}$ sensor direction is aligned with the targeted component. The second order needs $C^{20}$ sensor rotations and the sums of the measured fluxes. Thus, only two different and simple shapes allow identification of the first eight components of the multipolar expansion by filtering the other components up to the fifth order.

This new set of loops provides an accurate identification of $A_{n m}$ like the first sensor shown in Fig. 3. The relationships between measured fluxes and $A_{n m}$ components are given by

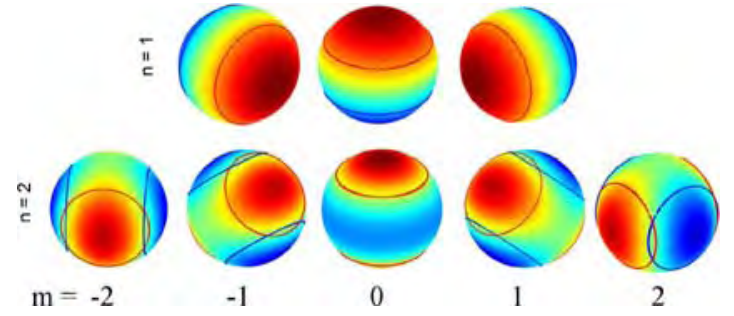

Fig. 7. Second sensor set using rotation properties.

$$
\left\{\begin{array}{l}
A_{1 m}=\frac{10^{8} r_{M}}{32 \pi} F_{l u x_{1 m}} \\
A_{20}=\frac{6125 \cdot 10^{4} r_{M}^{2}}{3 \pi \sqrt{21}} F_{l u x_{20}} \\
A_{2 m}=\frac{6125 \cdot 10^{4} r_{M}^{2}}{3 \pi \sqrt{21}}\left(\frac{1}{\sqrt{3}}\right) \text { Flux }_{2 m} \text { for } m \neq 0
\end{array}\right.
$$

where Flux $x_{n m}$ corresponds to the total flux through the sensor coils.

In Fig. 7, the complexity of the system is significantly reduced for the coils, but there are still too many coils to actually build the complete sensor. To alleviate this problem, we will limit the sensor design to four coils that can be rotated to capture the measurements, illustrated in [5]. However, it must be pointed out that the mechanics of rotation can lead to new difficulties.

\section{F. Validations}

The complete validation has been reached using mathematical (by finite element method) and experimental methods. The results have been presented previously [5].

\section{CONCLUSION}

We have shown that it is possible to improve the dipole component measurement of the Standard device. It is also possible to create simple coil systems that measure di- and quadripolar components by filtering both high orders (respectively up to the fifth order and up to the sixth order). The presented method improves on previously published results by simplifying the coil design.

Furthermore, our identification method is validated by experimental and mathematical results. In future work, we can focus on the construction of the full coil systems that include rotation.

\section{ACKNOWLEDGMENT}

The authors would like to thank D. Lyon for grammar and language assistance.

\section{REFERENCES}

[1] J. R. Bergervoet and H. Van Veen, "A large-loop antenna for magnetic field measurements," in Proc. Zurich Int. Symp. EMC, 1989, pp. 29-34.

[2] Standard CISPR 16-1, annexe P, pp. 230-237, 396-409, 2002.

[3] B. Vincent, O. Chadebec, J.-L. Schanen, C. A. F. Sartori, L. Krähenbühl, R. Perrussel, and K. Berger, "New robust coil sensors for near field characterization," J. Microw., Optoelectron. Electromagn.c Appl., vol. 8, no. 1, pp. 64S-77S, Jun. 2009.

[4] R. Green, "Spherical harmonic lighting: The gritty details," in Proc. Game Dev. Conf., 2003, pp. 1-47.

[5] B. Vincent, O. Chadebec, J.-L. Schanen, and K. Berger, "Loop antennas for near field multipolar expansion identification: First experimental validations," IEEE Trans. Instrum. Meas., ICONIC 2009-Special Issue, to be published. 